[0212-7199 (2005) 22: 10; pp 489-492] ANALES DE MEDICINA INTERNA Copyright $($ C 2005 ARAN EDICIONES, S.L.

AN. MED. INTERNA (Madrid) Vol. 22, N. ${ }^{\circ} 10$, pp. 489-492, 2005

\title{
Infección bacteriémica por Nocardia otitidiscaviarum: revisión a propósito de un caso
}

\author{
F. J. CANDEL, J. GONZÁLEZ1', M. MATESANZ1, R. CINZA' R. RÍAS, I. CANDEL ${ }^{1}$, \\ J. A. PONTES ${ }^{1}$, V. ROCA-ARBONÉS' ${ }^{1}$ J. J. PICAZO
}

Servicios de Microbiología Clínica y ${ }^{\prime}$ Medicina Interna. Hospital Clínico San Carlos. Madrid

\author{
BACTEREMIC INFECTION DUE TO NOCARDIA OTITIDISCAVIA- \\ RUM: CASE REPORT AND REVIEW
}

\section{RESUMEN}

Presentamos un caso de neumonía bacteremica causada por Nocardia otitidiscaviarum en un paciente con bronconeumopatia crónica obstructiva corticodependiente. Los cultivos de sangre y esputo en medios para micobacterias resultaron positivos y la identificación se realizó mediante la secuenciación de $16 \mathrm{~S}$ rDNA. En el presente artículo se realiza una revisión de conjunto sobre el agente etiológico y se practica el estudio de susceptibilidad de la cepa mediante la técnica de E-Test.

PALABRAS CLAVE: Inmunosupresión. Infecciones. Nocardia otitidiscaviarum. Bacteriemia. Antibióticos. E-Test.

\begin{abstract}
We present a case of bacteremic pneumonia caused by Nocardia otitidiscaviarum in a corticodependent COPD. Blood and sputum cultures on Mycobacterial media were positives and identification was done using $16 \mathrm{~S}$ rDNA sequencing. In this article we review the most relevant comunications about Nocardia spp infection and study the strain susceptibility using E-test.
\end{abstract}

KEY WORDS: Immunosupression. Infections. Nocardia otitidiscaviarum. Bacteremia. Antibiotics. E-Test.

Candel FJ, González J, Matesanz M, Cinza R, Cías R, Candel I, Pontes JA, Roca-Arbonés V, Picazo JJ. Infección bacteriémica por Nocardia otitidiscaviarum: revisión a propósito de un caso. An Med Interna (Madrid) 2005; 22: 489-492.

\section{INTRODUCCIÓN}

Las infecciones por Nocardia spp son relativamente frecuentes en pacientes inmunosuprimidos. Entre ellas, las causadas por Nocardia otitidiscaviarum son rara vez comunicadas. Presentamos el caso de una neumonía bacteriémica en un paciente EPOC de fatales consecuencias.

\section{CASO APORTADO}

Varón de 79 años, alérgico a betalactámicos con antecedentes personales de EPOC corticodependiente con varias reagudizaciones anuales y en tratamiento con oxigeno domiciliario y broncodilatadores a dosis plenas (situación funcional III-IV de NYHA). Acude al hospital por un cuadro de 3 días de evolución de tos con expectora- ción purulenta, mayor dificultad respiratoria y empeoramiento del nivel de conciencia. En la exploración física destacó fiebre de $38^{\circ} \mathrm{C}$, cianosis central y periférica, aumento de la presión venosa yugular, taquicardia a $120 \mathrm{lpm}$, alargamiento del tiempo espiratorio, sibilancias y roncus espiratorios en ambos campos pulmonares, sobre todo en el derecho. En el abdomen hepatomegalia dolorosa y edemas en ambas piernas. La radiografía de tórax mostró un aumento de densidad en lóbulo inferior derecho, de bordes mal definidos (Fig.1). En el hemograma se objetivaron 22.970 leucocitos $/ \mathrm{mm}^{3}\left(93^{\mathrm{N}}, 3,4^{\mathrm{L}}\right), \mathrm{Hb} 13$

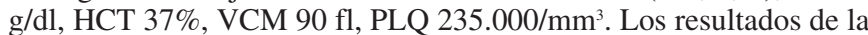
gasometría fueron $\mathrm{pH} 7.45, \mathrm{pO}_{2} 57, \mathrm{pCO}_{2} 36.4, \mathrm{HCO}_{3} 25,9, \mathrm{SatO}_{2}$ $90 \%$. Se practicaron cultivos de sangre y esputo en medios generales (agar sangre y chocolate, Biomerieux ${ }^{\circledR}$ ) y para el estudio de micobacterias [Bactec 960 MGIT (Mycobacterial Growth Indicator Tube) y Lowenstein (Becton Dickinson ${ }^{\circledast}$ ) \}. Las tinciones de Gram y Ziehl del esputo no ofrecieron orientación diagnóstica. En el cultivo de esputo a las 24 horas (calidad intermedia, > 25 leucocitos 10-25 células epi-

Trabajo aceptado: 26 de mayo de 2005

Correspondencia: F. J. Candel. Servicio de Microbiología Clínica. Hospital Clínico San Carlos. C/ Doctor Martín Lagos, s/n. 28040 Madrid. e-mail: fj.candel@terra.es 


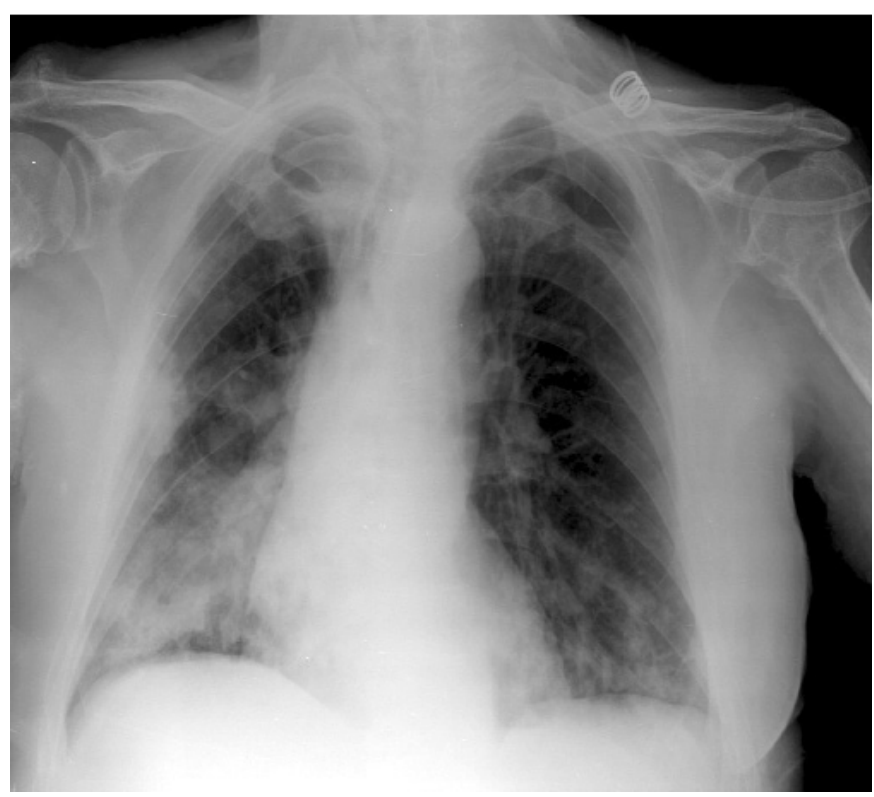

Fig. 1. La radiografía de tórax muestra un infiltrado difuso en lóbulo inferior derecho.

teliales) tan sólo se objetivó flora habitual del tracto respiratorio. Se inició tratamiento empírico con Levofloxacino e.v. (500 mg/día), Vancomicina e.v. $(1 \mathrm{~g} / 12 \mathrm{~h})$ y Tobramicina e.v. $(200 \mathrm{mg} / 12 \mathrm{~h})$. El paciente desarrolló un empeoramiento progresivo clínico (hipotensión, disnea y fiebre) y analítico (el hemograma entonces mostró 40.000 leucocitos $/ \mathrm{mm}^{3}$ ), falleciendo al cuarto día del ingreso.

El mismo día del fallecimiento se observó positividad tanto en el hemocultivo como en el cultivo de esputo, ambos en medio especifico para micobacterias (MGIT). La tinción de Ziehl de ambas mostró marañas de bacilos Ziehl positivos ramificados (Fig. 2A). Del pase del caldo de MGIT a medio de Mueller-Hinton (Biomerieux ${ }^{\circledast}$ ) y Lowenstein, crecieron a las 24 horas unas colonias secas, inicialmente blanquecinas, que a las 48 horas, viraban a asalmonadas, con tex- tura similar al yeso (Fig. 2B). Se envió la cepa problema al Centro Nacional de Microbiología de Majadahonda. Allí se amplificó por PCR la secuencia 16S rDNA del microorganismo y, con un 100\% de homologías, se identificó como Nocardia otitidiscaviarum.

\section{DISCUSIÓN}

Pautas terapéuticas en torno a $10 \mathrm{mg}$ diarios de prednisona durante tres semanas producen una supresión funcional prolongada de linfocitos T, una inhibición de las interleucinas 1-6 y un descenso del TNF. Todo esto favorece la aparición de infecciones oportunistas tales como viriasis, micosis invasivas e infecciones por bacterias intracelulares (Salmonella spp, Brucella spp, Legionella spp y Listeria spp), micobacterias y nocardias (1).

Nocardia spp es una bacteria grampositiva aerobia, perteneciente al orden actinomycetales. Produce fundamentalmente cuadros respiratorios (neumonía) o neurológicos (meningitis, absceso cerebral) en pacientes inmunodeprimidos. También es responsable de osteomielitis e infección posquirúrgica. Una de las series mas amplias comunicada sobre Nocardia spp en Europa, es la perteneciente al Instituto Pasteur (Paris) (2) que cifra su incidencia entre 150-250 nuevos casos de nocardiosis año en Francia. Las especie más frecuentemente aislada es $\mathrm{N}$ asteroides $(66,7 \%)$, seguida de $N$. farcinica $(23,8 \%)$. Nocardia otitidiscaviarum sólo representó el $5 \%$ de los aislamientos.

El determinante de patogenicidad más importante de Nocardia spp es la resistencia a la fagocitosis, evitando la inducción fagosoma-lisosoma y la acidificación del fagosoma. La situación de inmunosupressión celular provocada por los esteroides caracterizada por la linfopenia, descenso de las citoquinas y la inactivación de la fagocitosis, favorece el desarrollo intracelular del microorganismo. La presencia de leucocitosis y neutrofilia no es suficiente para resolver la infección, ya que son los linfocitos $\mathrm{T}$, deficientes en el tratamiento este-
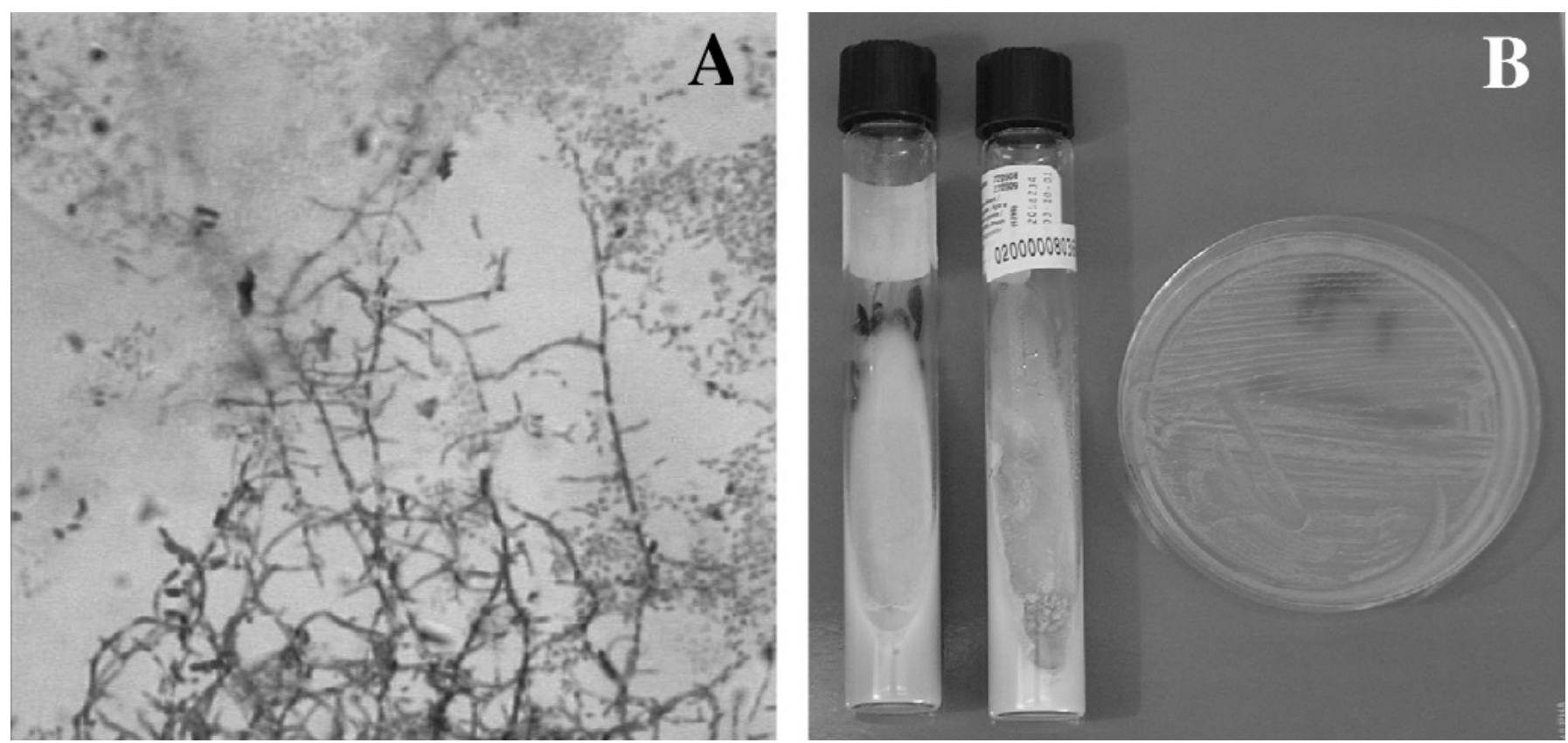

Fig. 2. Tinción de Ziehl modificada (Kinyoun) del cultivo positivo en MGIT. B: Nocardia spp. creciendo en medios de Lowenstein-Jensen y Müeller-Hinton. 
roideo, los principales elementos bactericidas capaces de evitar la diseminación de Nocardia spp (1).

La afectación pulmonar es la forma más frecuente de presentación de la infección por Nocardia spp. Acontece fundamentalmente en pacientes VIH o corticodependientes (EPOC, transplantados). Los síntomas más frecuentemente comunicados son la fiebre y el incremento de la disnea. Radiológicamente los pacientes pueden presentar condensación única o múltiple y la evolución clínica suele ser desfavorable, sobre todo en aquellos con enfermedad diseminada (3).

Se han comunicado infecciones invasivas por $N$ otitidiscaviarum causando abscesos cerebrales o pulmonares $(4,5)$ y neumonías nodulares en pacientes inmunosuprimidos, pero raramente ha sido aislada en hemocultivos (6). La bacteriemia por Nocardia spp es un entidad infrecuente, no habiéndose descrito más de 40 casos en toda la literatura (6-10). Ocurre principalmente en el contexto de malignidad, la prevalencia es mayor en hombres y precede al foco original en la mitad de los casos $(8,11)$. Se relaciona con la presencia de dispositivos intravasculares (sobre todo en pacientes transplantados), puede aparecer concomitantemente en el cultivo con otros patógenos (33\%), en su mayoría gramnegativos y su aparición suele indicar mal pronostico o preterminalidad (8). La especie mas frecuente aislada es $N$ asteroides complex y son mas raras $N$ nova, $N$ otitidiscaviarum y $N$ farcínica $(10,11)$. Se han comunicado un total de 5 nocardemias relacionadas con catéteres centrales ( $3 \mathrm{~N}$ asteroides, $1 \mathrm{~N}$ nova y $1 \mathrm{~N}$ otitidiscaviarum), 3 de ellas fueron aisladas en pacientes inmunosuprimidos u oncológicos. En ellos, el catéter fue retirado y los pacientes estuvieron en tratamiento antibiótico durante largo tiempo ( 2 o 3 meses). En los otros 2 pacientes sin enfermedad oncológica o inmunosupresora se mantuvo el catéter. Ambos fueron tratados con carbapenemes durante 14-21 días con buenos resultados $(7,12)$.

En la actualidad se emplean sistemas automáticos de identificación como ID32C o Micro-scan (RAI/HNID), técnicas de ribotipaje o análisis de restricción enzimática después de PCR, como se procedió con la cepa de nuestro paciente. Los estándares para el análisis de la sensibilidad de Nocardia spp son la microdilución en caldo o la difusión en disco, aunque existen técnicas recientes como el BACTEC radiométrico o el E-Test, que presentan buenas correlaciones con la microdilución y podrían ser utilizadas de rutina en el laboratorio clínico $(13,14)$.

Aunque la mayoría de las especies del género Nocardia spp son sensibles a combinaciones terapéuticas de Cotrimo- xazol, existen particularidades entre estas $(15,16)$. El grupo asteroides es altamente sensible a sulfonamidas (91-100\% de los aislados) y Minociclina (94-100\%), así como a betalactamicos, en especial a Imipenem (71-100\%) y cefalosporinas de tercera generación $(80 \%)$. También presenta sensibilidad frente a amikacina (80\%). El género farcinica es resistente a Gentamicina, Tobramicina y Kanamicina, pero es sensible a Amikacina (100\%). Presenta susceptibilidad disminuida frente a cefalosporinas de tercera generación (tan sólo $7 \%$ frente a cefotaxima) y sensibilidad intermedia para quinolonas (68-88\% de los aislados) y carbapenemes (Imipenem 64-87\%). Nocardia nova es sensible a sulfonamidas (89-97\%), aminoglucosidos (Amikacina 100\%), Minociclina (89-100\%), así como cefalosporinas de tercera generación y carbapenemes (100\% en ambas). Nocardia transvalensis y brasiliensis presenta también alta susceptibilidad a sulfonamidas (82-100\%). N. brasiliensis es sensible, además, a cefalosporinas de tercera generación (Cefotaxima 88-100\%), pero presenta susceptibilidad disminuida frente a carbapenemes (sólo sensibles el 20-30\% de los aislados). A N. transvalensis le sucede lo contrario, presenta alta susceptibilidad frente a carbapenemes y baja para las cefalosporinas de tercera generación $(50 \%)$. Nocardia otitidiscaviarum es resistente a sulfonamidas y sensible a minociclina y amikacina y presenta susceptibilidad disminuida frente a betalactamicos y carbapenemes.

Se evaluó en nuestra cepa la susceptibilidad a antimicrobianos empleando E Test. Para ello inoculamos la colonia en $5 \mathrm{ml}$ de caldo estéril de Mueller-Hinton. El caldo fue incubado a $35^{\circ} \mathrm{C}$ en atmósfera aerobia y vorteado hasta conseguir un crecimiento optimo y una disolución homogénea. Cuando la densidad óptica del caldo era de 1.0 McFarland, usando el colorímetro Vitek Densicheck (bioMerieux ${ }^{\circledR}$ ) se diluyó con suero fisiológico hasta conseguir un $0.5 \mathrm{McFarland}$. Esta dilución final se extendió sobre placas con Mueller-Hinton agar. Los E-Test se realizaron siguiendo las instrucciones del fabricante. Dos tiras equidistantes con distintos antibióticos fueron emplazadas en cada placa. Todas las placas fueron incubadas a $35^{\circ} \mathrm{C}$ en atmósfera aerobia, haciendo la lectura a las 24 y 48 horas en el punto de intersección de la elipse con la escala de CMI en la tira del E-Test. Los resultados se resumen en la (Tabla I). Los puntos de corte del National Committee of Clinical Laboratory Standars NCCLS (2000C) (17) han sido tomados de Tomlin et al (18).

Nuestra cepa era resistente a Cotrimoxazol y sensible a aminoglucosidos, betalactamicos y carbapenemes. El trata-

TABLA I

CMIS FRENTE A NOCARDIA OTITIDISCAVIARUM DETERMINADAS MEDIANTE E-TEST (18)

\begin{tabular}{|c|c|c|c|c|}
\hline & \multicolumn{2}{|c|}{$\mathrm{CM} /(\mu \mathrm{g} / \mathrm{ml})$} & \multicolumn{2}{|c|}{ Punto de corte $(\mu \mathrm{g} / \mathrm{ml})$} \\
\hline & 24 horas & 48 horas & $S$ & $R$ \\
\hline Trimetoprim-sulfametoxazol & 4 & 32 & $\leq 2 / 38$ & $\geq 8 / 152$ \\
\hline Imipenem & 0,50 & 1 & $<4$ & $>16$ \\
\hline Amoxicilina-clavulánico & 1,5 & 1,5 & $<8 / 4$ & $>16 / 8$ \\
\hline Levofloxacino & 1 & 1,5 & ND & ND \\
\hline Cefotaxima & 4 & 4 & ND & ND \\
\hline Amikacina & 0,75 & 0,75 & $<16$ & $>32$ \\
\hline
\end{tabular}

CMI: concentración mínima inhibitoria; S: sensible; R: resistente; ND: no datos en la bibliografía revisada. 
miento empírico elegido para este paciente era activo in vitro, pero el paciente empeoró y falleció. Hartmann et al (19) obtuvieron buenos resultados frente a esta especie en un paciente trasplantado combinando Meropenem y Rifampicina e.v durante 6 semanas, seguido de Ciprofloxacino y Rifampicina durante 2 meses. Matsuo et al (20) observaron mejoría en un paciente inmunocompetente combinando Claritromicina (600 mg/día) con Amikacina (400 mg/día). Por último, Linezolid ha demostrado actividad in vitro frente a varias especies de Nocardia spp (21) y ha sido comunicada una buena respuesta clínica con la combinación de Linezolid, Cefotaxima y Minociclina (22).

\section{AGRADECIMIENTOS}

Al Centro Nacional de Microbiología de Majadahonda (Madrid) por la identificación molecular de la cepa. A Ana Merino (enfermera y técnico de laboratorio), por su colaboración en la reproducción de los estándares de Tomlin et al en nuestro laboratorio.

\section{Bibliografía}

1. Cunha BA. Infecciones relacionadas con corticosteroides en las enfermedades reumatológicas. En: Picazo JJ, Bouza E. Infección 2002. 2002. Servisistem 2000 S.L: 177-88.

2. Boiron P, Provost F, Chevrier G, Dupont B. Review of nocardial infections in France 1987 to 1990. Eur J Clin Microbiol Infect Dis 1992; 11: 709-14.

3. Mari B, Monton C, Mariscal D, Luján M, Sala M, Domingo C. Pulmonary nocardiosis: clinical experience in ten cases. Respiration 2001; 68: $382-8$.

4. Hemmersbach-Miller M, Martel AC, Benítez AB. Brain Abscess due to Nocardia Otitidiscaviarum: report of a Case and Review. Scand J Infect Dis 2004; 36: 381-4.

5. Yoshida K, Bandoh S, Fujita J, et al. Pyothorax Caused by Nocardia Otitidiscaviarum in a Patient with Rheumatoid Vasculitis. Intern Med 2004; 43: 615-9.

6. Petersen DL, Hudson LD, Sullivan K. Disseminated Nocardia caviae with positive blood cultures. Arch Intern Med 1978; 138: 1164-5.

7. Kontoyiannis DP, Jacobson KL, Whimbey EE, Rolston KV, Raad II. Central venous catheter-associated Nocardia bacteremia: an unusual manifestation of nocardiosis. Clin Infect Dis 2000; 31: 617-8.

8. Kontoyiannis DP, Ruoff K, Hooper DC. Nocardia bacteremia. Report of 4 cases and review of the literature. Medicine (Baltimore) 1998; 77: 255-67.

9. Esteban J, Ramos JM, Fernández-Guerrero ML, Soriano F. Isolation of Nocardia sp. from blood cultures in a teaching hospital. Scand J Infect Dis. 1994; 26: 693-6.

10. Christidou A, Maraki S, Scoulica E, et al. Fatal Nocardia Farcinica Bacteremia in a Patient with Lung Cancer. Diag Microbiol Infect Dis 2004; 50: $135-9$.

11. Leederman, ER; Crum, NF. A Case Series and Focused Review of Nocardiosis: Clinical and Microbiologic Aspects. Medicine (Baltimore) 2004; 83: 300-313

12. Lui WYS, Lee ACW, Que TL. Central Venous Catheter-Associated Nocardia Bacteremia. Clin Infect Dis 2001; 33: 1613-4.
13. Biehle JR, Cavalieri SJ, Saubolle MA, et al. Comparative evaluation of the E-Test for susceptibility testing of Nocardia Species. Diagn Microbiol Infect Dis 1994; 19: 101-10.

14. Amaybe A, Kohner PC, Wollan PC, et al. Comparison of agar dilution, broth microdilution, disk diffusion, E-Test and BACTEC radiometric methods for antimicrobial susceptibility testing of clinical isolates of the Nocardia asteroides complex. J Clin Microbiol 1997; 35 : 847-52.

15. Brown BA, Wallace RJ. Nocardia farcinica: Un agente involucrado en infecciones nosocomiales y adquiridas en la comunidad. En: Bouza E, Picazo JJ. Infeccion 2001. 2001. servisistem 2000 S.L: 197-211.

16. Sorrell TC, Iredell JR, Mitchell DH. Nocardia species. In: Mandell GL, Bennett JG, Dolin R. Principles and practice of infectious diseases. 5th ed. Churchill Livinstone 2000; 2, Chp 244: 2637-44.

17. National Committee for Clinical Laboratory Standards (NCCLS) (2000c). M24-T2: susceptibility testing of Mycobacteria, Nocardia and other Actinomycetes; tentative standard-second edition. NCCLS. Wayne, PA.

18. Tomlin P, Sand C, Rennie RP. Evaluation of E test, disk diffusion and broth microdilution to establish tentative quality control limits and review susceptibility breakpoints for two aerobic Actinomycetes. Diagn Microbiol Infect Dis 2001; 40: 179-86.

19. Hartmann A, Halvorsen CE, Jenssen T, Bjorneklett IB, Bakke SJ, Hirschberg $\mathrm{H}$, et al. Intracerebral abscess caused by Nocardia otitidiscaviarum in a renal transplant patient cured by evacuation plus antibiotic therapy. Nephron 2000; 86: 79-83.

20. Matsuo K, Takeuchi M, Kawata N, Nabe M, Okamoto M, Tada S, et al. Pulmonary Nocardia otitidiscaviarum infection in a inmunocompetent host. Nihon Kokyuki Gakkai Zasshi 2000; 38: 844-9.

21. Brown-Elliot BA, Ward SC, Crist CJ, et al. In vitro Activities of Linezolid Against Multiple Nocardia Species. Antimicrob Agents Chemother 2001; 45: 1295-7.

22. Torres HA, Bhavananda TR, Raad II, et al. Nocardiosis in Cancer Patients. Medicine (Baltimore) 2002; 81: 388-97. 